\title{
Comparison of oligonucleotide-microarray and serial analysis of gene expression (SAGE) in transcript profiling analysis of megakaryocytes derived from CD $34^{+}$cells
}

\author{
Hyung-Lae Kim \\ Department of Biochemistry \\ College of Medicine \\ Ewha Womans University, Seoul, Korea \\ Tel, 82-2-2650-5727; Fax, 82-2-2652-7846; \\ E-mail, hyung@ewha.ac.kr
}

Accepted 7 October 2003

Abbreviations: SAGE, serial analysis of gene expression

\begin{abstract}
For the comprehensive analysis of transcript expression, the array-based hybridization analysis and the serial analysis of gene expression (SAGE) are commonly used platforms. The SAGE is based on a high-throughput sequencing of ditags derived from the transcript. DNA microarrays are a powerful tool for monitoring thousands of transcripts simultaneously, whereas the Genechip (Affimatrix microarray) technology is based on the hybridization of a single probe or other manufacturer's microarrays (cDNA- or oligonucleotide-microarray) procedures include the competitive hybridization of two probes. In this study, the quantitative accuracy of expression using oligonucleotide-microarray was determined by comparing data set from the SAGE. In previous study the microSAGE was performed for the megakaryocytes and nonmegakaryocytes derived from human cord blood CD $34^{+}$cells by ex vivo expansion using thrombopoietin, and a total of 38,909 tags representing 8,976 unique genes were obtained. On the identical RNA, expression profiling was also carried out using oligonucleotide-microarray (MAGIC II $10 \mathrm{~K}$ chip, Macrogen). The most frequently expressed genes in human megakaryocytes were identified as platelet factor 1 followed by annexin $A 1$, ribosomal protein $\mathbf{S 2 3}$. The majority of the 50 most highly expressed genes in the CD $34^{+}$-derived megakaryocytes were those involved in protein synthesis, e.g., ribosomal proteins. The expression level through the single channel of oligonucleotide-microarray and SAGE have a fairly good cor-
\end{abstract}

relation in terms of absolute analyses and that the correlation is higher for the genes with higher expression levels.

Keywords: oligonucleotide microarray; transcript expression analysis

\section{Introduction}

For the analysis of the global gene expression, several methods such as DNA microarray and serial analysis of gene expression (SAGE) were developed. DNA microarrays are technology to profile the expression of thousands of transcripts simultaneously (Brown and Botstein, 1999; Lockhart and Winzeler, 2000; Noordewier and Warren, 2001), and two different types of microarray technologies are available; Genechips (Affymetrix platform) and spotted microarrays. The Genechips produced by photolithography and hybridize the single cRNA probe have certain advantages over spotted microarray, such as that crosshybridization could be avoided, quality control by sequence validation of PCR clones is not required, and noncompetitive nature of hybridization make small amount of RNA sample detectable (Kane et al., 2000; Lockhart and Winzeler, 2000). As this platform is not accessible for every laboratory, spotted microarrays of longer oligonucleotides (50-70 mer) are becoming attractive and alternative platform (Hughes et al., 2001).

The spotted microarrays are manufactured by spotting the cDNAs or synthetic oligonucleotides to the glass plate. Oligonucleotide-microarrays were recently preferred over cDNA microarray due to the easiness of target gene design and the reliability of hybidization (Southern et al., 1999). Oligonucleotide-libraries covering large parts of the transcriptome of several organisms are now available from many companies. Quantitation of transcripts using oligonucleotide-microarrays is based on the competitive hybridization of each labeled CRNA probes to the oligonucleotides anchored on the glass. In short, the total RNA from two different samples was reverse-transcribed into cDNAs, which were labeled with fluorescent dyes Cy3 (blue) and Cy5 (red). The cDNA (or cRNA) labeled with Cy3 and $\mathrm{Cy} 5$ are mixed together and hybridized against oligonucleotides on the same array. The two populations compete for the same targets or probe spots on 
the array. The spot intensity at the two wavelengths is determined. A ratio or log ratio between the two fluorescent intensities is calculated. The most advantage of the microarray platform in global analysis of gene expression is the speed of data aquisition, but the gene with low level of expression would not be discriminated from that of no expression.

SAGE is a high throughput sequencing based technique used to estimate the relative expression levels of thousands of transcripts by sequencing concatamers of short sequence tags derived from transcript sample. SAGE developed by Velculescu is based on the principle that a nucleotide sequence of 9-10 bases (a gene tag) at a specific location within a transcript represents a specific gene (Velculescu et al., 1995). These tags, which contain sufficient information to identify a gene uniquely, are generated, concatenated and cloned. By sequencing the clones, the identification and the abundance of a transcript are established. The number of tags for a given sequence by the total tags gives the absolute abundance of the tag. It allows for the establishment of both a representative and comprehensive different gene expression profile in various cell types and organs under physiological and pathological states. The data set by SAGE platform has been used construct the transcript expression databases (http://www.sagenet.org).

It has been a great concern whether the expression analysis using microarray has quantitative accuracy, and whether it has reliable correlation comparing SAGE data set. Although microarray technology has previously shown to be correlated well with other analytical methods including SAGE, but the microarray technology used was always the Affymetrix Genechip based on the single probe hybridization (Ishii et al., 2000; Evans et al., 2002). As the other microarray procedures adopted the competitive nature the hybridization of two probes, they have been applied to evaluate only the relative changes of expression. This necessitates the evaluation of the competitive hybridization-based microarray using two probes by comparing other platforms of global transcript analysis. The accuracy of spotted microarray in comparion with SAGE has not been done. Previously, we have performed microSAGE in megakaryocytes and non-megakaryocytes derived from human $\mathrm{CB} C D 34^{+}$cells by ex vivo expansion using thrombopoietin (Kim et al., 2002). Total 38,909 tags representing 8,976 unique genes were obtained. In this study, the quantitative accuracy of oligonucleotide-microarray (MAGIC II chip; Macrogen) was determined by comparing transcript profile to that of SAGE method on the identical mRNA specimens. From this study it is observed that the gene expression levels from oligonucleotide-microarray fairly correlated well with the SAGE for the moderate- to high expressed transcripts.

\section{Materials and Methods}

\section{Preparation of cells and $m R N A$}

The procedures used to prepare samples are described previously (Kim et al., 2002). Briefly, cord blood (CB) was obtained from full-term deliveries with informed consent. Cord blood mononuclear cells were isolated by centrifugation on a FicollHypaque (density, 1.077; APB, Upsalla, Sweden) density centrifugation. The CD $34^{+}$cell fraction was positively isolated using an anti-CD34 monoclonal antibody (QBEND 10; Miltenyi Biotech, Bergisch Gladbach, Germany) The CD $34^{+}$cells were cultured in serum-free essential media supplemented with bovine serum albumin, insulin, and transferrin (StemCell Technologies, Vancouver, BC, Canada), and were stimulated with recombinant human TPO (50 ng/ml; Kirin Brewery, Maebashi, Japan) alone. After 10 days, megakaryocytes fraction was separated from non-megakaryocytes fraction using an anti-CD61 (GPIlb/Illa) monoclonal antibody (Dako, Copenhagen, Denmark). Purity of each separated fraction was verified by flow cytometry with a different antibody reacting with megakaryocytes (FITCconjugated anti-human CD41; BD). Total RNA from these cells was isolated using TRIZOL (Gibco BRL, $N Y$ ) according to the manufacturer's instructions. The same RNA was used in both protocols.

\section{SAGE protocol}

The microSAGE protocol has been described previously (Datson et al., 1999; Kim et al., 2002). Biotinylated oligo dT primer annealed $10 \mu \mathrm{g}$ of total RNA was converted to cDNA with a cDNA synthesis kit (Gibco BRL) in streptavidine coated PCR tube (Roche; Mannheim, Germany; http://biochem.boehringermannheim.com). The cDNA was cleaved with Nla III, and was ligased to the oligonucleotide containing recognition sites for $B s m F$ I. After ligation, the bound CDNA was released from the matrix by digestion with BsmF I. SAGE tag overhangs were filled with Klenow enzyme, and tags from two pools were combined and ligated to each other. The ligation product was amplified by PCR, concatemerized and cloned into the Sphl site of pZero-1 (Invitrogen, Calsbad, CA). Clones were sequenced with the BigDye terminator kit and analyzed using $\mathrm{ABI} 3,700$ automated sequencer (PerkinElmer, Branchberg). Sequence files were analyzed by means of SAGE analysis software version 4.12. Sequence files were analyzed, and statistical analysis of the data was performed by the use of SAGE software, version 4.12 (courtesy of Victor Velculescu and Ken Kinzler, Johns Hopkins University School of Medicine) (Velculescu, Zhang et al., 1995). The identity of the mRNAs corresponding to the SAGE tags was determined through inspection and comparison 
with the SAGEmap (www.ncbi.nlm. nih.gov/SAGE/SAGEtag.cgi) and UniGene (www.ncbi.nIm.nih.gov/UniGene) databases. SAGE tags that had no reliable matches and multiple matches in Unigene clusters were excluded from the list. Mitochondrial genes and ESTs were also omitted. If more than one gene tag corresponded to the same Unigene cluster, then the higher ranked tag was selected.

\section{Microarray protocol}

Experimental procedures for microarray were performed according to the Macrogen MAGIC II-10K technical Manual. The identical total RNA as in the SAGE protocol was used to generate cDNA. Total RNA $(5 \mu \mathrm{g})$ was converted into double stranded cDNA using the cDNA synthesis system (Roche) using T7$(\mathrm{dT})_{24}$ primer. The each cDNA was purified using RNeasy kit (Qiagen, Valencia, http://www.qiagen.com). Non-megakaryocytes selected by CD61 antibody were used as reference in the experiment. Each Cy3-(for non-megakaryocytes), or Cy5-(for megakaryocytes) labeled cRNA was synthesized using the Megascript T7 kit (Ambion, Austin), using Cy3-CTP and Cy5-CTP (APB, Uppsala Sweden). The cRNA was cleaned using RNeasy (Qiagen). Labeled $15 \mu \mathrm{g}$ of each cRNA was mixed and fragmented by heating to $94^{\circ} \mathrm{C}$ for 15 min. Fragmented CRNA was hybridized with MAGIC II-10 K microarray (Macrogen, Seoul, Korea) for 16 $\mathrm{h}$ at $42^{\circ} \mathrm{C}$. Arrays were then washed and scanned with an Array scanner (APB). Acquired images were processed and analyzed statistically for interpretation of analyzed spot intensity results using Imagene v4.1 software (Roche). Non-biological factors that may contribute to variability of data were minimized using global normalization/scaling with data from all probe sets, and normalization between the microarrays was also carried out. Each chip contains a total of 10,368 elements of which 10,108 are unique genes/clusters. The length of oligonucleotides was 50 -mer.

\section{Results}

Total of 38,909 SAGE tags representing 8,976 unique genes were obtained from megakaryocytes. After normalization of fluorescence intensities from the microarray, 8,889 transcripts were used for the exact comparison to that of SAGE using Unigene accession number. Resulting total 1,168 transcripts were matched to each system. For the CD $34^{+}$-derived megakaryocytes, the top 50 transcripts in terms of cy5 fluorescence intensity were listed in Table 1. The most frequently expressed genes in human megakaryocytes were identified as platelet factor 1 followed by annexin $A 1$, ribosomal protein $S 23$. The majority of the 50 most highly expressed genes in the CD $34^{+}$- derived megakaryocytes were those involved in protein synthesis, e.g., ribosomal proteins.

The correlation between the probe intensity observed in oligonucleotide-microarray and the actual mRNA abundance in terms of absolute analyses was shown in Figure 1. Comparing the logarithmic scale of the fluorescent intensities and tag frequencies for the 1,168 Unigenes, the Spearman's coefficient is $0.425(P=0.0001$, Figure $1 \mathrm{~A})$. Oligonucleotide-microarray intensity scores are one or two orders of mag
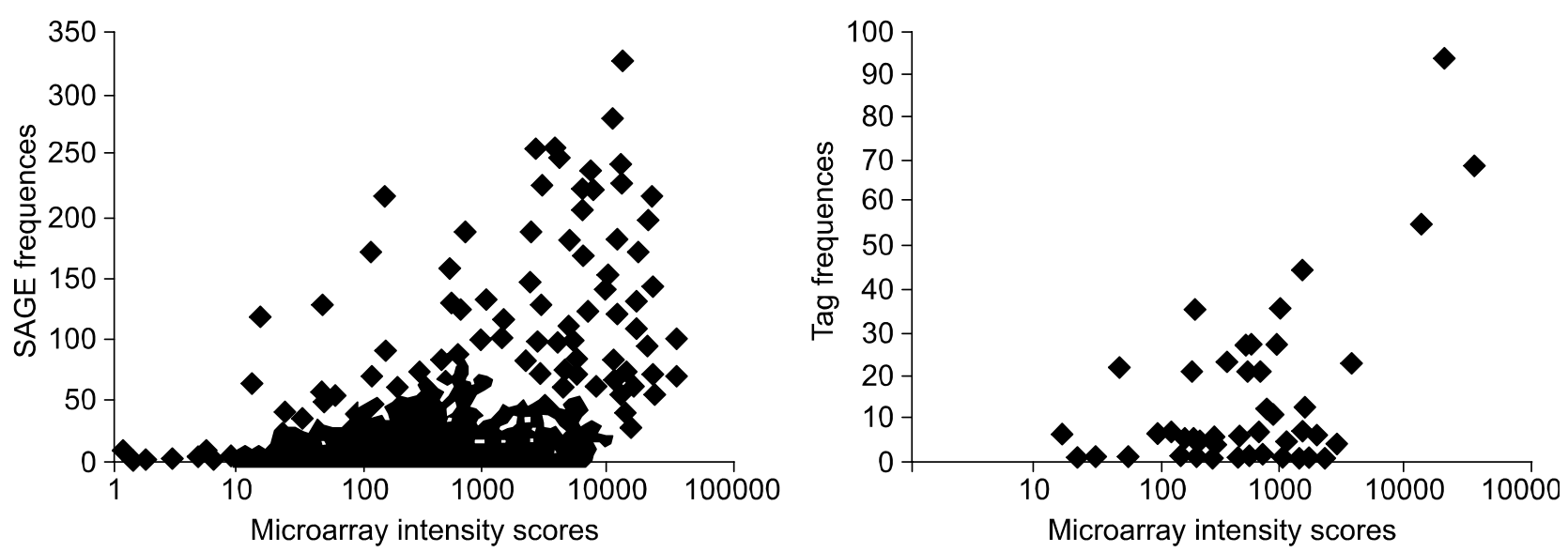

Figure 1. Scatter graph of intensity values in oligonucleotide-microarray and the number of tags in SAGE. Intensity scores and the number of tags are plotted in a logarithmic scale on the abscissa and the ordinate, respectively. Genes with no tag and tags that match multiple genes were excluded. GeneChip scores are one or two orders of magnitude higher than SAGE frequencies. (A) For the CD34 ${ }^{+}$-derived megakaryocytes, the coefficient was as high as $0.425(n=1,168, P<0.0001)$. (B) For the transcript without any significant change (expression fold-changes $<2)$ in microarray, the coefficient was as high as $0.657(n=73, P<0.0001)$. 
Table 1. Transcript profile in $\mathrm{CD}_{61} 1^{+}$megakaryocytes derived from $\mathrm{CD} 34^{+}$cells.

\begin{tabular}{|c|c|c|c|}
\hline Intensity & UniGene & Description & Tags \\
\hline 38,457 & Hs.81564 & plateletfactor 4 & 69 \\
\hline 35,717 & Hs.75510 & annexinA11 & 117 \\
\hline 22,942 & Hs.3463 & ribosomalprotein $\$ 23$ & 218 \\
\hline 22,083 & Hs.73797 & G protein alpha 15 ( $\mathrm{Gq}$ class) & 72 \\
\hline 21,200 & Hs.2164 & pro-plateletbasic protein & 94 \\
\hline 17,021 & Hs.184014 & ribosomalprotein L31 & 172 \\
\hline 16,453 & Hs.76847 & alphaglucosidase II alpha subunit & 130 \\
\hline 16,160 & Hs.418072 & ferritin,heavy polypeptide 1 & 62 \\
\hline 14,599 & Hs.54673 & Tumornecrosis factor superfamily, member 13 & 65 \\
\hline 14,023 & Hs.169238 & fucosyltransferase 3 & 30 \\
\hline 13,543 & Hs.380843 & ribosomalprotein S6 & 227 \\
\hline 13,391 & Hs.76686 & glutathioneperoxidase 1 & 55 \\
\hline 13072 & Hs.389335 & ribosomalprotein $\mathrm{L} 13 \mathrm{a}$ & 329 \\
\hline 12,817 & Hs. 406511 & ribosomalprotein, large, P0 & 242 \\
\hline 12,552 & Hs.168383 & intercellularadhesion molecule 1 (CD54), & 118 \\
\hline 11,381 & Hs.182740 & ribosomalprotein S11 & 33 \\
\hline 10,977 & Hs.301636 & peroxisomalbiogenesis factor 6 & 83 \\
\hline 10,926 & Hs.434029 & ribosomalprotein $\mathrm{S} 12$ & 283 \\
\hline 9,778 & Hs.432645 & red cell anion exchanger 3 ' non-coding region & 153 \\
\hline 9,230 & Hs.301547 & ribosomalprotein S7 & 142 \\
\hline 7,696 & Hs.334807 & ribosomalprotein $\mathrm{L} 30$ & 235 \\
\hline 7,647 & Hs.234518 & ribosomalprotein L23 & 222 \\
\hline 7,442 & Hs.430150 & ferritin,light polypeptide & 21 \\
\hline 7,277 & Hs.409045 & ribosomalprotein L6 & 124 \\
\hline 6,536 & Hs.406682 & ribosomalprotein L26 & 124 \\
\hline 6,458 & Hs.426460 & ribosomalprotein L9 & 222 \\
\hline 6,190 & Hs.65588 & DAZassociated protein 1 & 168 \\
\hline 5,541 & Hs.235422 & ribosomalprotein L14 & 27 \\
\hline 5,415 & Hs.412900 & ribosomalprotein L10 & 47 \\
\hline 5,410 & Hs.169476 & glyceraldehyde-3-phosphatedehydrogenase & 6 \\
\hline 5,338 & Hs.419463 & ribosomalprotein $\mathrm{L} 23 \mathrm{a}$ & 23 \\
\hline 5,288 & Hs. 5174 & ribosomalprotein $\mathrm{S} 17$ & 41 \\
\hline 5,215 & Hs.180450 & ribosomalprotein $\mathrm{S} 24$ & 82 \\
\hline 5,188 & Hs.334842 & tubulin,alpha, ubiquitous & 39 \\
\hline 5,183 & Hs.397609 & ribosomalprotein $\mathrm{S} 16$ & 74 \\
\hline 5,032 & Hs.256184 & eukaryotictranslation elongation factor 1 gamma & 100 \\
\hline 5,027 & Hs.5308 & ubiquitinA-52 residue ribosomal protein fusion product 1 & 23 \\
\hline 4,769 & Hs.326249 & ribosomalprotein L22 & 110 \\
\hline 4,531 & Hs.426035 & RAN,member RAS oncogene family & 35 \\
\hline 4,520 & Hs.14376 & actin,gamma 1 & 62 \\
\hline 4,324 & Hs.169793 & ribosomalprotein L32 & 74 \\
\hline 4,108 & Hs.283781 & musclespecific gene & 21 \\
\hline
\end{tabular}


Table 1. Continued.

\begin{tabular}{cllr} 
Intensity & UniGene & \multicolumn{1}{c}{ Description } & Tags \\
\hline 4,079 & Hs.431927 & ribosomalprotein L21 & 249 \\
3,793 & Hs.279652 & mitochondrialribosomal protein L4 & 23 \\
3,767 & Hs.48516 & beta-2-microglobulin & 41 \\
3,731 & Hs.10306 & naturalkiller cell group 7 sequence & 97 \\
3,715 & Hs.356371 & ribosomalprotein L28 & 256 \\
3,691 & Hs.233936 & myosinregulatory light chain & 23 \\
3,526 & Hs.115808 & leukocyte-associatedlg-like receptor 1 & 23 \\
3,410 & Hs.76067 & heatshock 27kDa protein 1 & 6 \\
\hline
\end{tabular}

RU, resonance unit; RF, rheumatoid factor; ESR, erythrocyte sedimentation rate; ND, not done 1:100 diluted joint fluids were injected onto the CM5 sensor chips followed by the running buffer. Specific binding signals were obtained by subtraction of nonspecific signals from binding on BSA-immobilized flow cells. Laboratory test results were shown.

nitude higher than SAGE frequencies due to amplification of RNA. Genes ranked high by the SAGE method generally showed high-intensity scores in microarray analysis. This graph suggests that the two analytical procedures, oligonucleotide-microarray and SAGE, have a fairly good correlation in terms of absolute analyses and that the correlation is higher for genes with higher expression levels.

Conventionally using the spotted-microarray, the further analysis has been carried out for the transcript with the expression fold-changes greater than 2 . Transcripts without any significant change and the fold-changes less than 2 were excluded. Figure $1 \mathrm{~b}$ shows the scatter graph of the log intensity values in oligonucleotide-microarray and the SAGE tag numbers. The coefficient became as high as 0.657 ( $n=73, P<0.001)$. The scatter graph of fold changes in both analytical procedures indicates that the comparative analyses have a better correlation among those genes with high SAGE tags, but have a relatively poor correlation among genes with lower SAGE tags. The relatively poor correlation in the range of low tag numbers may be attributable to the limited number of tags caused by extensive exclusion of genes from the list.

\section{Discussion}

The spotted-microarray was used for the study of transcriptional activity only in terms of fold changes. So this makes the spotted-microarray uneasy to compare the each fluorescent intensities of microarray with the expression level of other platforms, such as SAGE. Theoretical basis of using the intensity from the single channel of cy5 (or cy3 as well) in comparing SAGE frequencies is as follows. The amount of fluorescence-labelled cRNA used for hybridization on microarray is about $20 \mu \mathrm{g}$. If the frequency of the RNA for the moderately expressed species is $1 / 3,000$, its amount would be around 10 fmole (Rininge et al., 2000). As $50 \mathrm{fmol}$ of oligonucleotides were overlaid for each spot on the microarray, the amount would be more than that of the moderate to low expressed transcripts. So, it is supposed that the hybridization mechanism would not be so competitive in the experimental condition using two probes, excluding a couple of the highly-expressed transcripts.

As a whole, spotted oligonucleotide-microarray technology is reasonably reliable for the analysis of expression profiling of genes with higher expression levels and greater changes in expression by SAGE and the MAGIC II chip The coefficient was 0.42, which is somewhat lower than that of the other report using GeneChip (Ishii et al., 2000). The GeneChip technology, which is based on the single probe hybridization, was described to be moderately quantitative in terms of comparative analyses (Evans et al., 2002). When compared for the transcript with expression-fold changes greater than 2 , the coefficient was 0.67 . If the transcripts with low tag numbers were excluded, the coefficient would be higher. The detection efficiency of microarray for the transcripts with the low tag numbers (for example 1 or 2 tags) was highly variable, as 0 to $100 \%$ (Evans et al., 2002). So the reduction of correlation was mainly ascribe to the irrelevance between the low SAGE tags and microarray fluorescence intensities. The detailed causes might come from the limitations and drawbacks of each technology.

Microarray is a high throughput method for profiling gene expression but many variables influence the outcome of the experiment. It may under perform due to probe design issues, such as; distance of the tar- 
get sequence from the poly A tail; secondary structures with the target sequence; variations in pin geometry; leading to different amounts of DNA hybridizing within different non-specific hybridization; variations in exposure of different portions of the slide to the hybridization solution; efficiencies in dye incorporation; and cross-reactivity of the probe with other transcripts, each of which may influence detection (Schuchhardt, et al., 2000). Since there are a large number of factors that contribute to experimental error and biological variation, replicate experiments are needed to normalize the data (Lee et al., 2000).

On the other hand, SAGE may under-perform because specific transcripts may be missed due to the absence of a recognition site for the anchoring enzyme or CG-content bias (Margulies et al., 2001). In addition, incorrect tag counts can arise from incomplete digestion or alternative polyadenylation, giving rise to multiple tags derived from a single transcript. Moreover, some of the transcript can be matched multiply. To increase tag length, $R s a \mathrm{I}$ and $\mathrm{Bsm} \mathrm{FI}$ were often used in SAGE library construction. That would generate $14 \mathrm{bp}$ tags (Ryo et al., 2000), together with GTAC (Rsa I site sequence), total $18 \mathrm{bp}$ stretch should be conveniently used for matching Unigene library. Also, sequencing errors, especially in the population of tags encountered only once, can contribute to the differences between SAGE and MAGIC II chip data. In this study, the number of gene tags analyzed was not sufficient to cover the entire set of mRNAs in a single cell type. Therefore, statistical significance is not robust for those genes expressed at a low level. Namely, even when 300,000 tags were analyzed, there was a $92 \%$ chance of detecting a tag for transcripts when the expression on average was at least three copies per cell (Zhang et al., 1997).

The fold-changes of expression megakaryocytes/ non-megakaryocytes were comprared. The correlation could not be observed between two platforms, but the pattern in terms of increasing or decreasing was kept (data not shown). The overall expression fold-changes by MAGIC II chip had tendency of high value than that by SAGE. The causes of such pattern might be from the high incorporation efficiencies of Cy5-UTP, and the preference of the Cy5-labelled probes in the hybridization procedure. The higher quantum yield of the Cy5 fluorophore might also be contributed (Gruber et al., 2000; t Hoen et al., 2003).

This is the first report showing their quantitative analyses in spotted microarray using competitive hybridization. This study suggested that the expression intensities from a single channel of oligonucleotidemicroarray technology reliable to detect mediumto-high abundant transcripts in absolute expression analyses, and would be appropriate as a platform to build a gene expression database, but the determi- ning the fold-changes of expression needs other technologies of transcript analysis.

\section{Acknowledgement}

This study was supported by a grant of the Korea Health 21 R\&D Project, Ministry of Health \& Welfare, Republic of Korea (HMP-00-CH-04-0004).

\section{References}

Brown PO, Botstein D. Exploring the new world of the genome with DNA microarrays. Nat Genet 1999;21:33-7

Datson NA, van der Perk-de Jong J, van den Berg MP, de Kloet ER, Vreugdenhil E. MicroSAGE: a modified procedure for serial analysis of gene expression in limited amounts of tissue. Nucleic Acids Res 1999;27:1300-7

Evans SJ, Datson NA, Kabbaj M, Thompson RC, Vreugdenhil E, De Kloet ER, Watson SJ, Akil H. Evaluation of Affymetrix Gene Chip sensitivity in rat hippocampal tissue using SAGE analysis. Serial Analysis of Gene Expression. Eur J Neurosci 2002;16:409-13

Gruber HJ, Hahn CD, Kada G, Riener CK, Harms GS, Ahrer W, Dax TG, Knaus HG. Anomalous fluorescence enhancement of Cy3 and cy3.5 versus anomalous fluorescence loss of Cy5 and Cy7 upon covalent linking to $\operatorname{lgg}$ and noncovalent binding to avidin. Bioconjug Chem 2000;11:696-704

Hughes TR, Mao M, Jones AR, Burchard J, Marton MJ, Shannon KW, Lefkowitz SM, Ziman M, Schelter JM, Meyer MR, Kobayashi S, Davis C, Dai H, He YD, Stephaniants SB, Cavet G, Walker WL, West A, Coffey E, Shoemaker DD, Stoughton R, Blanchard AP, Friend SH, Linsley PS. Expression profiling using microarrays fabricated by an ink-jet oligonucleotide synthesizer. Nat Biotechnol 2001;19:342-7

Ishii M, Hashimoto S, Tsutsumi S, Wada Y, Matsushima K, Kodama T, Aburatani H. Direct comparison of GeneChip and SAGE on the quantitative accuracy in transcript profiling analysis. Genomics 2000;68:136-43

Kane MD, Jatkoe TA, Stumpf CR, Lu J, Thomas JD, Madore SJ. Assessment of the sensitivity and specificity of oligonucleotide (50mer) microarrays. Nucleic Acids Res 2000; 28:4552-7

Kim JA, Jung YJ, Seoh JY, Woo SY, Seo JS, Kim HL. Gene expression profile of megakaryocytes from human cord blood CD $34^{+}$cells ex vivo expanded by thrombopoietin. Stem Cells 2002;20:402-16

Lee ML, Kuo FC, Whitmore GA, Sklar J. Importance of replication in microarray gene expression studies: statistical methods and evidence from repetitive cDNA hybridizations. Proc Natl Acad Sci USA 2000;97:9834-9

Lockhart DJ, Dong H, Byrne MC, Follettie MT, Gallo MV, Chee MS, Mittmann M, Wang C, Kobayashi M, Horton H, Brown EL. Expression monitoring by hybridization to highdensity oligonucleotide arrays. Nat Biotechnol 1996;14:167580

Lockhart DJ, Winzeler EA. Genomics, gene expression and 
DNA arrays. Nature 2000;405,827-36

Margulies EH, Kardia SL, Innis JW. Identification and prevention of a GC content bias in SAGE libraries. Nucleic Acids Res 2001;29:E60

Noordewier MO, Warren PV. Gene expression microarrays and the integration of biological knowledge. Trends Biotechnol 2001;19:412-5

Rininger JA, DiPippo VA, Gould-Rothberg BE. Differential gene expression technologies for identifying surrogate markers of drug efficacy and toxicity. Drug Discov Today 2000; 5:560-8

Ryo A, Kondoh N, Wakatsuki T, Hada A, Yamamoto N, Yamamoto M. A modified serial analysis of gene expression that generates longer sequence tags by nonpalindromic cohesive linker ligation. Anal Biochem 2000;277:160-2
Schuchhardt J, Beule D, Malik A, Wolski E, Eickhoff $H$, Lehrach $H$, Herzel $H$. Normalization strategies for cDNA microarrays. Nucleic Acids Res 2000;28:E47

Southern E, Mir K, Shchepinov M. Molecular interactions on microarrays. Nat Genet 1999;21(supple 1):5-9

t Hoen PA, de Kort F, van Ommen GJ, den Dunnen JT. Fluorescent labelling of cRNA for microarray applications. Nucleic Acids Res 2003;31:e20

Velculescu VE, Zhang L, Vogelstein B, Kinzler KW. Serial analysis of gene expression. Science 1995;270:484-7

Zhang L, Zhou W, Velculescu VE, Kern SE, Hruban RH, Hamilton SR, Vogelstein B, Kinzler KW. Gene expression profiles in normal and cancer cells. Science1997;276:126872 